\title{
Nach langer Aufarbeitung endlich Klarheit
}

\author{
Jürg Schädelin ${ }^{a}$, Felix Nigglib, Hansruedi Völklec \\ a Dr. med., ehemaliger medizinischer Leiter der Abteilung Medikamentensicherheit und Epidemiologie bei Novartis \\ ${ }^{\text {b }}$ Prof. Dr. med., Leiter Kinderonkologie am Universitäts-Kinderspital Zürich \\ c Prof. Dr., Universität Fribourg, ehemaliger Leiter der Sektion Überwachung der Radioaktivität im BAG
}

Vor 30 Jahren publizierte die COMARE ihren ersten Report zu der festgestellten Häufung von Kinderleukämien um die Nuklearanlage von Sellafield. Ende des vergangenen Jahres ist der 17. Report* erschienen, der die ganze Aufarbeitung dieses Vorfalles zusammenfasst und aktualisiert. Die anfängliche Beurteilung eines fehlenden Zusammenhanges zwischen Nuklearanlage und Leukämie hat sich durch den weiteren Verlauf und Untersuchungen mit neu entwickelten Methoden noch erhärtet: Die radioaktiven Emissionen können nicht für die Leukämieerkrankungen verantwortlich gemacht werden

\begin{abstract}
Die COMARE (Committee on Medical Aspects of Radiation in the Environment) wurde als beratende Kommission des englischen Gesundheitsministeriums nach der Feststellung einer achtmal erhöhten Leukämiehäufigkeit in der bei der Nuklearanlage Sellafield liegenden Kleinstadt Seascale im Nordwesten Englands ins Leben gerufen und hat seither vorzügliche Arbeit bei der Aufklärung und Beratung zum Strahlungsrisiko der Bevölkerung geleistet.
\end{abstract}

\section{Die Geschichte einer Sensation}

Am 1. November 1983 strahlte ein lokaler Fernsehsender die Reportage Windscale: The nuclear laundry aus, in der berichtet wurde, dass in der nordenglischen Kleinstadt Seascale Kinderleukämien 10-mal häufiger auftreten als im Landesdurchschnitt, vermutlich als

\section{Enfin de la clarté}

II y a 30 ans, le Committee on Medical Aspects of Radiation in the Environment (COMARE) publiait son premier rapport sur l'accumulation constatée de cas de leucémie infantile autour de la centrale nucléaire de Sellafield. Le $17^{\mathrm{e}}$ rapport, qui résume et actualise l'ensemble du traitement de cet épisode, a été publié à la fin de l'année dernière. La conclusion initiale d'absence de lien entre la centrale et l'incidence des leucémies a encore été étayée par le déroulement ultérieur et les études basées sur de nouvelles méthodes: les émissions radioactives ne peuvent être tenues responsables des cas de leucémie.
Folge radioaktiver Emissionen der naheliegenden Wiederaufbereitungsanlage Sellafield. Ein Aufschrei der Entrüstung war die Folge, zumal fast 30 Jahre früher an diesem Ort ein Reaktorbrand zu grossflächiger Kontamination geführt hatte, deren Folgen allerdings dank korrekter Schutzmassnahmen minimiert werden konnten. Die damals geübte Informationspolitik war aber weitgehend auf Verschwiegenheit ausgerichtet gewesen, was schon daran exemplifiziert werden kann, dass die Nuklearanlage durch Umtaufen von Windscale in Sellafield von Makel befreit wurde.

Dies liess sich 25 Jahre später nicht mehr praktizieren. Zur Aufarbeitung der Vorwürfe setzte die Regierung eine prominent besetzte Fachkommission ein, die zwar durch einige Korrekturen an der Analyse das Ausmass der Gefährdung herunterschraubte, eine unerwartete Häufung dieser Krankheit aber bestätigte. Sie stellte aber auch fest, dass die gemessenen Strahlendosen in der Umgebung um Grössenordnungen zu tief lagen, als dass sie nach den damaligen Kenntnissen der Strahlenbiologie für einen derartigen Effekt verantwortlich gemacht werden könnten. Die Kommission empfahl eine weitere Überwachung der Situation unter Einschluss anderer Nuklearanlagen, was zur Gründung der COMARE als ständiges Fachgremium führte, das bis zum heutigen Tage diese Aufgabe kompetent wahrnimmt.

Weltweit wurden solche Untersuchungen auch für die weitaus zahlreicheren Kernkraftwerke durchgeführt und inzwischen in mehr als 100 Publikationen veröf- 
fentlicht. Die Ergebnisse sind schwer zusammenzufassen, da sich über den langen Zeitraum die Erhebung der statistischen Daten und die Prognose der Krankheit erheblich verändert hatten und vielfach inkompatible Kriterien und Methoden angewendet wurden. Eine geringere, aber vergleichbare statistische Häufung konnte jedoch nur für die militärische Anlage in Dounreay im Norden Schottlands und für das Kernkraftwerk Krümmel in Deutschland zweifelsfrei verifiziert werden.

Der Ende 2016 erschienene 17. Report der COMARE fasst die gesamte Aufarbeitung der Auffälligkeiten um Sellafield zusammen und ist geeignet, dieser Problematik ihre Schärfe zu nehmen.

\section{Die Situation hat sich epidemiologisch normalisiert}

Das Auftreten von Kinderleukämien wurde kontinuierlich verfolgt und wiederholt nach Massgabe der vermuteten Hypothesen für diese Häufung spezifisch ausgewertet. Die erhöhte Inzidenz konzentrierte sich auf den Zeitraum von 1979 bis 1984, und seit 1991 ist in der näheren Umgebung von Seascale keine typische

\section{Seit 1991 ist in der näheren Umgebung von Sellafield keine typische Kinderleukämie mehr diagnostiziert worden.}

Kinderleukämie mehr diagnostiziert worden. Seit nun 25 Jahren liegt die Häufigkeit von bösartigen Tumoren in den Landkreisen um Sellafield wie schon die ganze Zeit in den übrigen Regionen von Cumbria unter dem englischen Landesdurchschnitt. Dasselbe ist zu berichten von der Wiederaufbereitungsanlage von Dounreay

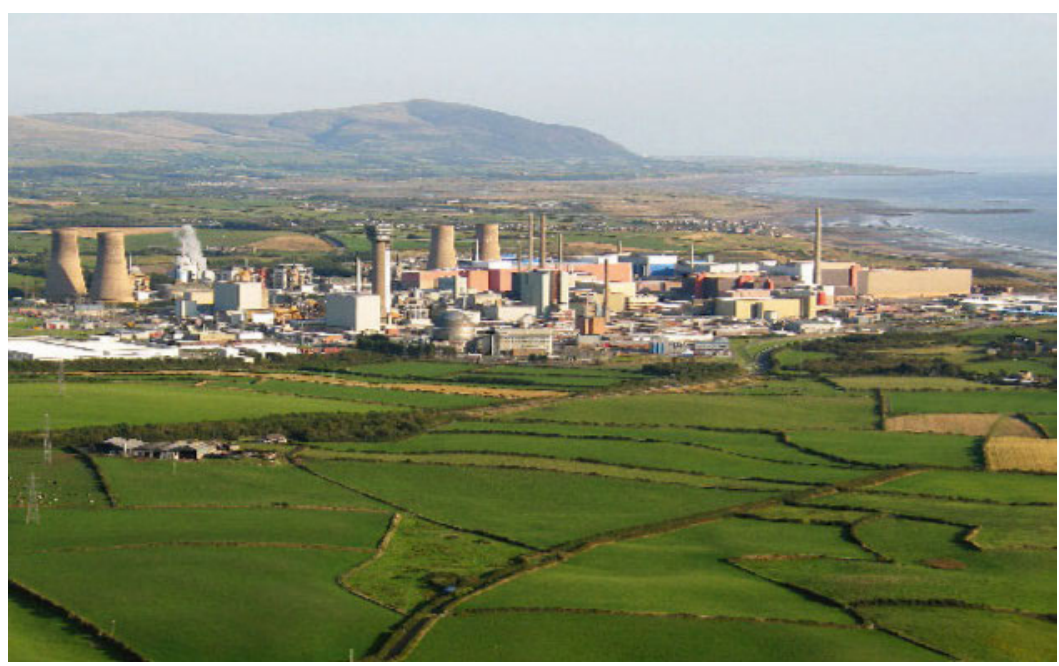

Die Nuklearanlage von Sellafield stand in den 80 er Jahren im Verdacht, eine Häufung von Kinderleukämien zu verursachen. an der Nordküste Schottlands, wo kurz nach Sellafield eine ähnliche, aber statistisch marginale Überzahl festgestellt worden war.

Alle vermuteten Ursachen, meist ein Verdacht aus einer explorativen Fallkontrollstudie, konnten epidemiologisch entkräftet werden: Beschäftigung der Väter in der Nuklearanlage, vorgeburtliche Strahlenexposition der Mütter oder Verzehr von potentiell kontaminierten Fischen erhöhten das Risiko nicht. In einem grossen Kohortenvergleich zwischen allen in Seascale geborenen und den dort die Schule besuchenden Kindern fanden sich die Erkrankten eher unter den Schülern, weniger unter den dort geborenen Kindern. Offensichtlich sind die nach der Geburt zugezogenen Kinder vermehrt erkrankt, die vor dem Schuleintritt weggezogenen Kinder dagegen weniger.

\section{Transparente Buchhaltung der abgegebenen Radioaktivität}

Die Kommission hat sich auch die Mühe genommen, alle dokumentierten und geschätzten Freisetzungen von Radioaktivität nach den neuesten mathematischen Modellen zu einer Gesamtstrahlenbelastung der Altersgruppe von 0 bis 24 Jahre zusammenzurechnen. Dies erwies sich als aufwendig wegen der ständig ändernden Funktion der Nuklearanlage (PlutoniumHerstellung von 1950 bis 1957, Brennstoff-Wiederaufbereitung von 1952 bis 1973 und Betrieb experimenteller Reaktoren seit 1975). Über den ganzen Zeitraum verblieb die Strahlenbelastung durch natürliche Radioaktivität (kosmische Strahlung und terrestrische Isotope) als bedeutendste Strahlenquelle. Während in den frühen Jahren bis 1970 die Abgaben ins Meer einen relevanten Beitrag zur Strahlenbelastung ausmachten, nimmt in jüngerer Zeit die medizinische Anwendung die zweite Position ein, nur temporär übertroffen durch den Fallout aus Atombombentests und der Kontamination durch Tschernobyl. Wo sich einzelne Isotope durch das Gammaspektrum in der Umgebungsmessung identifizieren liessen, wurde die Quelle gesucht und beispielsweise Polonium 210 in einer nahegelegenen Düngemittelfabrik infolge Aufarbeitung von Phosphatmineralien gefunden.

Auch die Auswirkungen des Reaktorbrandes von Windscale im Jahr 1957 wurden retrospektiv erneut studiert. Dort wurde in bedeutendem Ausmass Jod 131 freigesetzt, allerdings etwa 1000-mal weniger als in Tschernobyl. Die Strahlenbelastung der Schilddrüse erreichte weder in der Umgebung noch in der Ausbreitungszone über Nordostengland bedenkliche Werte, dies vor allem wegen des sofort ausgesprochenen Verbotes, frische Milch zu trinken. Selbst episodenhafte kleine 
Vorfälle wurden von dieser Kommission bearbeitet: Eine zufällig festgestellte, zwar erhöhte, aber ungefährliche Radioaktivität auf einem einzelnen Grundstück in Seascale konnte auf den Taubenschlag eines Züchters zurückgeführt werden, dessen Vögel sich in einem stillgelegten und als Abfalllager benützen Gebäude des Werkgeländes tummelten. Das Anbringen von Drahtgittern erwies sich als eine wirksame und billige Massnahme zur Eindämmung dieser Kontamination.

Zusammenfassend belegt dieser Bericht erneut und überzeugender als zuvor, dass Nuklearanlagen, wie sie heute betrieben werden, nicht ein Risiko für Leukämien und Krebserkrankungen in der Umgebung darstellen. Eine dokumentierte, zusätzliche Strahlenbelastung fand sich in früheren Jahrzehnten, ohne dass die groben Mortalitätsdaten damals gehäufte Leukämien andeuteten. Die Behauptung, dass die Empfindlichkeit von Kleinkindern auf genetische Auswirkungen von ionisierenden Strahlen mehr als 100-mal grösser sei, wie es zur Erklärung der Sellafield-Epidemie nötig wäre, lässt sich durch keinerlei experimentelle Daten belegen. Die wahrscheinlichste Erklärung für die an beiden Orten unzweifelhaft festgestellte, temporäre Häufung von Kinderleukämien bleibt die im Gefolge der Bauarbeiten und des Betriebes massive Zuwanderung von Familien mit Kindern aus spärlich bewohnten Gebieten der Umgebung, die in der Schule und damit spät in der Entwicklung einer Vielzahl von ungewohnten Infektionserregern ausgesetzt waren, so dass deren Immunantwort in Einzelfällen fehlgeleitet wurde. In ähnlichen demographischen Konstellationen zeigte sich vielerorts eine $\mathrm{Zu}$ nahme der Kinderleukämien, wobei die Bevölkerungsbewegung durch ganz unterschiedliche Gründe und ohne vermutete Strahlenrisiken hervorgerufen worden war. Da Populationsexperimente für diese Fragestellung nicht machbar sind, wird erst die Aufdeckung und das Studium eines plausiblen biologischen Mechanismus diese Hypothese untermauern können.

\section{Vorbildlicher Umgang mit Risiko- situationen}

Über die lange Zeit ihres Wirkens hat sich die COMARE $\mathrm{zu}$ einem Kompetenzzentrum zur Beurteilung von Strahlenrisiken in der Allgemeinbevölkerung entwickelt. Durch ihren breit umschriebenen Auftrag und als permanente Institution war sie in der Lage, sich allen neu auftauchenden Fragen zu stellen, und hat oft auch neue Strahlungsrisiken publik gemacht und

\section{Alle vermuteten Ursachen konnten epidemiologisch entkräftet werden.}

quantifiziert. So beispielsweise die massive Zunahme der medizinischen Untersuchungen durch Computertomographie oder die bedenkenlos genutzten Solarien. Vor allem bemerkenswert ist aber die aufwendige und sorgfältige Berichterstattung, die sich nicht auf Information der Auftraggeber in der Administration und Politik beschränkte, sondern in breit gestreuten, fachlich fundierten und allgemeinverständlichen Reports ein breiteres Publikum ansprach. Wir können von den Engländern lernen, wie derartige Risikosituationen studiert und verfolgt werden sollten. Ausgewiesene Expertengruppen, die auf lange Sicht die Verantwortung übernehmen, die Situation zu analysieren und zu verfolgen, sind gehalten, die Überlegungen und Empfehlungen den Entscheidungsträgern wie auch den interessierten Bürgern verständlich darzulegen. Wie in jeder politisch heiklen Frage tauchen immer wieder selbsternannte «Experten» auf, und es fällt Laien und Politikern schwer, ihre Kompetenz kritisch zu beurteilen. Aber es sollte ihnen leicht fallen, ihre Ratgeber zu einer öffentlichen Berichterstattung zu verpflichten. Die Fachkollegen werden die Beurteilung ganz sicher

\section{Wir können von den Engländern lernen,} wie derartige Risikosituationen studiert und verfolgt werden sollten.

kommentieren und die Schlussfolgerungen kritisch hinterfragen. Und eine Verantwortung über einen längeren Zeitraum ist das beste Mittel, von Experten ausgewogene Stellungnahmen zu erhalten, da sie sich zwangsläufig mit ihrem früheren Urteil konfrontiert sehen. Nur auf diese Weise lassen sich übereilte Entscheide vermeiden und können andauernde Unklarheiten - wie in dieser Problematik die Befunde beim Kernkraftwerk Kümmel - aufgearbeitet und beantwortet werden.

\section{Bildnachweis}

Simon Ledingham, CC BY-SA 2.0, https://commons.wikimedia.org/w/ index.php?curid=7938296 